\title{
Responsible Artificial Intelligence as a Secret Ingredient for Digital Health: Bibliometric Analysis, Insights, and Research Directions
}

\author{
Samuel Fosso Wamba ${ }^{1}$ (iD $\cdot$ Maciel M. Queiroz ${ }^{2,3}$ (D) \\ Accepted: 3 May 2021 \\ (C) The Author(s), under exclusive licence to Springer Science+Business Media, LLC, part of Springer Nature 2021
}

\begin{abstract}
With the unparallel advance of leading-edge technologies like artificial intelligence (AI), the healthcare systems are transforming and shifting for more digital health. In recent years, scientific productions have reached unprecedented levels. However, a holistic view of how AI is being used for digital health remains scarce. Besides, there is a considerable lack of studies on responsible AI and ethical issues that identify and suggest practitioners' essential insights towards the digital health domain. Therefore, we aim to rely on a bibliometric approach to explore the dynamics of the interplay between $\mathrm{AI}$ and digital health approaches, considering the responsible AI and ethical aspects of scientific production over the years. We found four distinct periods in the publication dynamics and the most popular approaches of AI in the healthcare field. Also, we highlighted the main trends and insightful directions for scholars and practitioners. In terms of contributions, this work provides a framework integrating AI technologies approaches and applications while discussing several barriers and benefits of AI-based health. In addition, five insightful propositions emerged as a result of the main findings. Thus, this study's originality is regarding the new framework and the propositions considering responsible AI and ethical issues on digital health.
\end{abstract}

Keywords Artificial intelligence $\cdot$ Machine learning $\cdot$ Digital health $\cdot$ Responsible AI $\cdot$ Bibliometric analysis

\section{Introduction}

With the emergence and adoption of leading-edge technologies (Alahakoon et al., 2020; Beydoun et al., 2019; Queiroz et al., 2020a; Sivarajah et al., 2017), mostly artificial intelligence (AI) (Alahakoon et al., 2020; Duan et al., 2019; Dwivedi et al., 2019; Fosso Wamba et al., 2021), society, organizations, and people are experimenting with unprecedented changes in their lives. In this context, one of the most promising areas that $\mathrm{AI}$ can remodel is related to healthcare systems (He et al., 2019; Jiang et al., 2017; Rajkomar et al.,

Maciel M. Queiroz

maciel.queiroz@docente.unip.br

Samuel Fosso Wamba

s.fosso-wamba@tbs-education.fr

1 Information, Operations and Management Sciences, TBS Business School, 1 Place Alphonse Jourdain, 31068 Toulouse, France

2 Paulista University - UNIP, Postgraduate Program in Business Administration, Dr. Bacelar Street 1212, 04026-002 Sao Paulo, Brazil

3 School of Engineering, Mackenzie Presbyterian University, Consolação Street 930, 01302-000 Sao Paulo, Brazil
2018; Vayena et al., 2018). From the patient and medical sides, AI promises to reshape the patients' journey and experience while providing unprecedented support to medical practices (He et al., 2019; Jiang et al., 2017) and personalized medicine.

Against this background, the literature exploring the interplay between AI in healthcare systems contexts increased significantly in the last years (Aisen et al., 1997; Benight et al., 2013; Chen \& Asch 2017; Klinker et al., 2019; Perry et al., 2007; Rajkomar et al., 2019; Stephanie \& Sharma, 2020). In this vein, different approaches and perspectives of AI usage in medicine activities evolved considerably. For instance, robots for surgery assistance (Mukherjee \& Sinha 2020), disease prediction assisted by machine learning (Jadhav et al., 2019), exoskeletons applications in rehabilitative assistance (Perry et al., 2007), healthcare robots for older's user's assistance (Broadbent et al., 2009), among others.

More recently, with mobile phones' mass diffusion, different healthcare systems' processes were digitalized, thus enabling electronic health systems (e-health) (Klinker et al., 2019; Rajkomar et al., 2018) and enhancing collaboration between patients, physicians, nurses, and other healthcare professionals. Moreover, while more and more robots are getting 
specialized in surgery activities (Kim 2014), there is an unprecedented increase in the processing power of computers, all of which adds to other AI techniques aimed to unlock the whole potential of AI in medicine. For example, machine learning (Chen and Asch 2017; Deo 2015; Jadhav et al., 2019), deep learning (De Fauw et al., 2018; Esteva et al., 2019; Miotto et al., 2017), big data (that uses different AI approaches) (Ching et al., 2018; Mukherjee, 2020; Obermeyer et al., 2016; Wang et al., 2018), have been used successfully to improve the patients' journey and experience, as well as medical practices. Thus, it can be seen that AI approaches enable more accurate and personalized medicine (Gottlieb et al., 2011; Rajkomar et al., 2019).

From this standpoint, smart technologies as applied to personalized medicine (Mukherjee, 2020), especially with the use of AI techniques and approaches, are disrupting traditional healthcare systems through different digitalizing processes (Feldman et al., 2020; Klinker et al., 2019; Mukherjee, 2020; Sharma \& Kshetri 2020; Stephanie \& Sharma, 2020). In other words, they are giving rise to new possibilities for the patient's treatment and medical activities. For instance, telemedicine (Serrano et al., 2020) increased without precedents, especially during the COVID-19 epidemic outbreak (Queiroz et al., 2020b). On the one hand, healthcare systems are becoming more digital (Klinker et al., 2019; Sharma \& Kshetri 2020). On the other hand, ethics and responsible practices in healthcare systems (Wang et al., 2020) seem to become a huge challenge to address, especially by using AI approaches.

Although the healthcare transformation literature to more digital health (Klinker et al., 2019) is emerging, the literature reporting the healthcare systems and their variations (digital health, digital healthcare, e-health, etc.) is steadily eluding a digital perspective, mainly taking into account the responsible $\mathrm{AI}$ and ethical perspective. Also, there is a substantial gap when considering the interplay between artificial intelligence approaches and digital health contexts based on a bibliometric analysis aiming to consolidate the literature. Also, the responsible AI that refers to its integration with ethical aspects on AI usage, enabling more transparency and privacy in AI applications by the organizations, is a still nascent topic (Wang et al., 2020) applied in the healthcare domain toward the digital health. Besides, little is known about the key AI-related technologies, their applications, benefits, and barriers in the healthcare systems towards digital health. Therefore, to understand in-depth this nascent field (responsible AI in digital health), a longitudianl exploration of the AI literature on related domains is fundamental. The extant literature concerning AI-related technologies in healthcare systems are predominantly focused on robotics for assist surgery (Esteva et al., 2019), predictions approach (Jadhav et al., 2019), electronic record (Rajkomar et al., 2018), among others. However, considering the responsible $\mathrm{AI}$ and the ethical issues on digital health seems an unexplored research stream (Wang et al., 2020).

In this context, this study explores the extant literature about artificial intelligence approaches on healthcare systems, considering the digital perspective (digital health, digital healthcare, e-health, etc.), and, more specifically, the responsible AI and the ethical issues in the digital health domain. Thus, our study is guided by the following research questions:

- What is the publication dynamics on the interplay between $\mathrm{AI}$ and healthcare systems?

- How is artificial intelligence being used in digital health systems?

- What are the main trends regarding artificial intelligence in digital health systems?

- What are the main limitations and ethical concerns about responsible artificial intelligence usage for digital health systems?

Our study brings unique insights and contributions by identifying the AI's main dynamics applied in healthcare systems and its journey through the digital health systems. It then reports the main trends, the most popular AI approaches, barriers, and benefits related to AI in digital health contexts. Besides, insightful research directions are proposed for scholars, practitioners, and decision-makers interested in exploring and gaining a deeper understanding of digital health and the role of AI as a responsible and disruptive cutting-edge technology. Furthermore, this work's originality resides in the novel framework to practitioners and scholars gain a more indepth understanding of the responsible $\mathrm{AI}$ in the digital health domain. Also, we provide five propositions derived from the framework that scholars can empirically validate it.

The paper is organized as follows. In Section 2, we introduce the bibliometric approach and protocol used. In sequence, in Section 3, we provide the main findings, including the frequency of publication of papers per year, the most relevant sources and countries, the most cited papers, trend topics, among others. Section 4 is dedicated to the discussion, contributions, implications, research agenda, and limitations. Finally, in Section 5, we present the final remarks and conclusions of this work.

\section{Bibliometric Approach}

Bibliometric analysis is a powerful approach to understanding a specific field's dynamics and behavior by providing different metrics (Beydoun et al., 2019; Kapoor et al., 2018; Mishra et al., 2018; Peng et al., 2017). In this regard, we followed the best practices related to lead a reliable bibliometric analysis (Beydoun et al., 2019; Nobre \& Tavares 2017) of the artificial intelligence approach in healthcare contexts. In view of this, first, we found one of the trustworthy and leading databases 
(Web of Science) (Clarivate Analytics 2020). Second, we performed a research protocol (Fig. 1). Although we did not restrict the year range in the search, it appears that the first paper retrieved dated back to 1977. Third, for results analysis, we employed the VOSviewer (Mishra et al., 2018) and Biblioshiny (Aria \& Cuccurullo 2017), which are specialized software applications to conduct a bibliometric analysis. Finally, we elected prominent categories to show the dynamics, trends, hot topics, and research directions to the field. Thus, variations on this related-type of strategy have been successfully used by the recent literature (Fosso Wamba et al., 2021; Queiroz et al., 2020b).

\section{Findings from the Bibliometric Analysis}

As mentioned previously, our search did not restrict a particular range of years, but the first paper retrieved appeared only in 1977 (Table 1). Of 14,469 retrieved papers, 341 presented incomplete information to the bibliometric analysis. Thus, we considered 14,128 papers from 51,458 authors. It should be pointed that out of 14,128 documents, only $8.49 \%$ (1200) were single-authored. Also, we can see a large number $(25,100)$ of keywords provided by the authors.

\subsection{Documents Frequency Over the Years}

Considering the publication of the documents over the years, Fig. 2 points out the frequency. We can see four different periods. In the first period, from 1977 to 1990, the maximum number of documents published in a year was 6 documents (1987) - the second period went from 1991 to 2006. We can see consistent growth, exceeding 100 documents published in
Table 1 Main information about the data collection

\begin{tabular}{ll}
\hline Description & Results \\
\hline Main information about data & \\
Timespan & $1977: 2020$ \\
Sources (Journals, Books, etc.) & 5174 \\
Documents & 14,128 \\
Average years from publication & 4.33 \\
Average citations per documents & 10.95 \\
Average citations per year per doc & 1.973 \\
Document contents & \\
Keywords Plus (ID) & 14,310 \\
Author's Keywords (DE) & 25,100 \\
Authors & \\
Authors & 51,458 \\
Author Appearances & 75,645 \\
Authors of single-authored documents & 1063 \\
Authors of multi-authored documents & 50,395 \\
Authors collaboration & \\
Single-authored documents & 1200 \\
Documents per Author & 0.281 \\
Authors per Document & 3.56 \\
Co-Authors per Documents & 5.23 \\
Collaboration Index & 3.80 \\
\hline
\end{tabular}

2006. In the third period (2007 to 2014), the publications outperformed 120 per year, achieving 419 in 2014. From 2015 to 2020, we can see unprecedented growth, breaking the milestone of 500 publications a year. Also, in 2019 and 2020, the output on these topics outperformed 2900 and 3900 documents per year, respectively. In this vein, this exceptional

Fig. 1 Research protocol

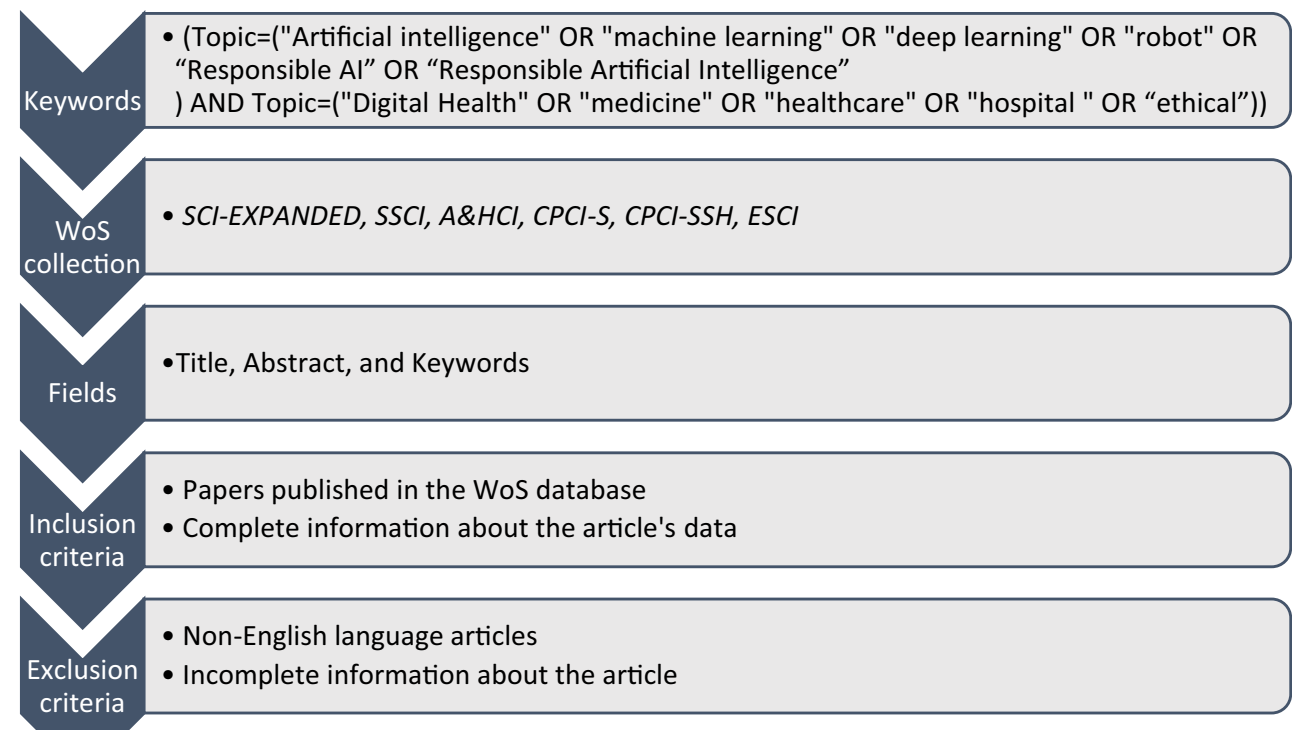


growth can be justified by the emergence and popularity of different $\mathrm{AI}$ techniques and computational power.

\subsection{Top 20 Sources}

Considering the most relevant sources based on the output, Table 2 shows the top 20 and indicates that IEEE Access was the only journal that virtually reached 300 papers. Besides, we can see that the journals dedicated to medical issues and medical informatics dominate the list. Also, no traditional journals from other fields like information systems, operations management, and operations research appeared in the top 20 ranks.

\subsection{Top 20 Most Cited Countries}

Regarding the most cited countries, Table 3 underlines the top 20 ranks, based on total citations. Only the USA, China, and the United Kingdom reached more than 10,000 citations. In addition, the USA got virtually 5 times $(63,009$ citations) the number of citations from China $(12,991)$ and the United Kingdom (12,821 citations). Italy completes the top 5 (8,229), and Korea $(6,628)$. Although the USA was topping the chart, the table highlights that countries from Europe and Asia achieved great participation. Furthermore, Oceania countries (Australia and New Zealand) obtained expressive participation. Unfortunately, countries from underrepresented regions (Latin America and Africa) did not appear in the ranking. Surprisingly, based on the average citations per article, Slovenia was ranked first (28.974), followed by New Zealand (19.082), the Netherlands (16.133), Belgium (15.883), and the USA (15.808).

\subsection{Top 20 Most Cited Documents}

Table 4 draws our attention to the most cited documents, including the authors, sources, the respective number of citations, and the citation per year. In this sense, we observe that two papers from the top 3 are relatively recent papers (published in 2016 and 2019). The top-ranked paper integrated big data and machine learning in the context of clinical medicine. In the papers of the top 5, learning (machine and deep) and robotics approaches were the most popular techniques used. In general, machine learning, robotics, and deep learning seem to feature among the most popular AI techniques in the top 20 papers. Furthermore, based on total citation per year (TCY), the top 3 papers were recent papers Topol (2019), De Fauw et al. (2018), and Obermeyer et al. (2016). Among them, the paper exploring the interplay between AI and humans in medicine, published in 2019, accrued an incredible number of citations per year (188.3). Another paper highlighted deep learning as a welcome technological development for retinal disease diagnosis, accounting for 119.5 per year. The third paper of the package was dedicated to integrating big data and machine learning in clinical medicine. Other recent papers (published in 2018) is achieving good performance, then suggesting the popularity of these topics on healthcare systems. Finally, it can be noted that no repeated author appeared as the first author in the top 20 papers and that journals

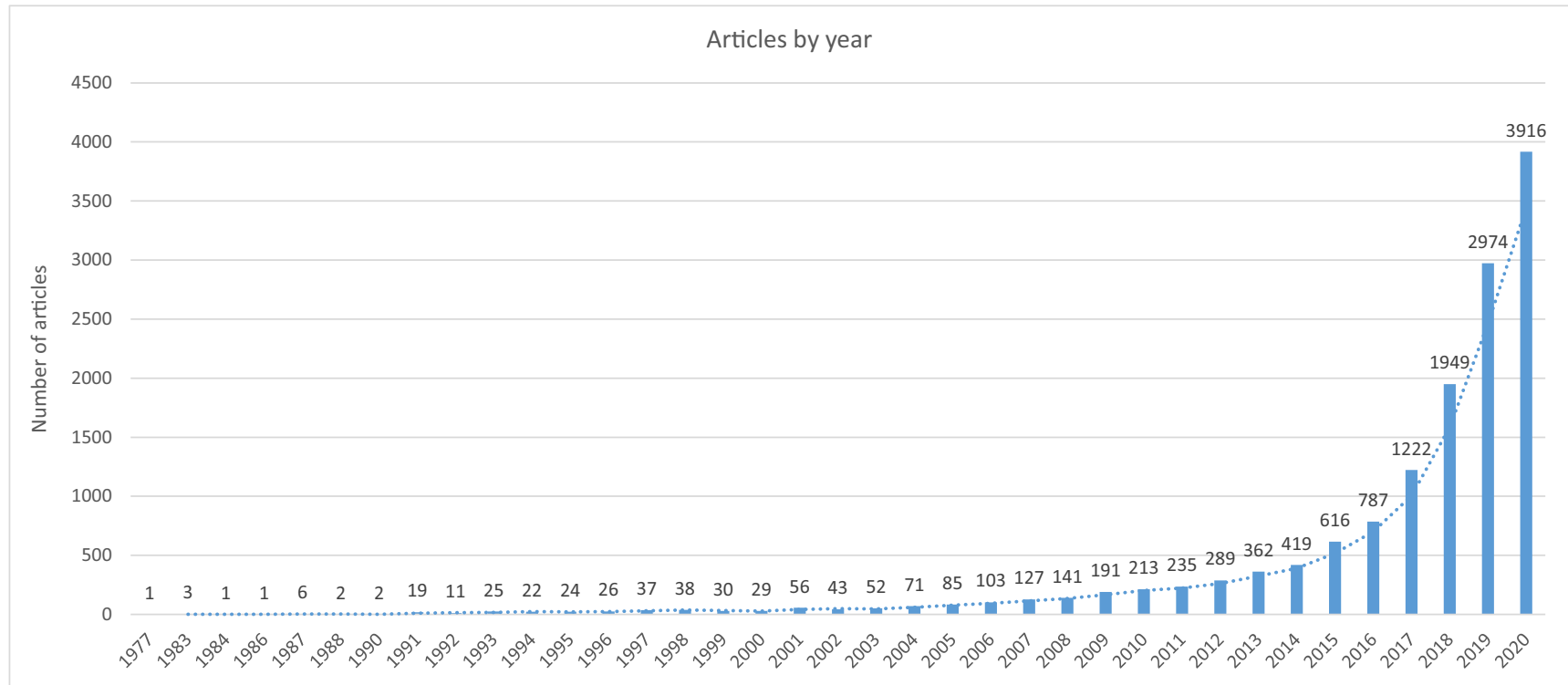

Fig. 2 Frequency of articles published between 1977 and 2020 
Table 2 Most relevant sources

\begin{tabular}{llc}
\hline Rank & Sources & Articles \\
\hline 1 & IEEE Access & 292 \\
2 & Journal of Endourology & 145 \\
3 & PLOS One & 144 \\
4 & Medical Physics & 130 \\
5 & Artificial Intelligence in Medicine & 129 \\
6 & Surgical Endoscopy and other Interventional Techniques & 126 \\
7 & Sensors & 114 \\
8 & Journal of Biomedical Informatics & 111 \\
9 & Journal of Medical Internet Research & 102 \\
10 & Scientific Reports & 102 \\
11 & BJU International & 97 \\
12 & Journal of the American Medical Informatics Association & 84 \\
13 & BMC Medical Informatics and Decision Making & 81 \\
14 & Applied Sciences-Basel & 77 \\
15 & International Journal of Medical Informatics & 66 \\
16 & European Urology & 65 \\
17 & IEEE Journal Of Biomedical and Health Informatics & 64 \\
18 & International Journal of Medical Robotics and Computer Assisted Surgery & 63 \\
19 & BMJ Open & 60 \\
20 & Journal of Robotic Surgery & 60 \\
\hline & &
\end{tabular}

specialized in medicine and nature subjects dominate the list.

Table 3 Most cited countries

\begin{tabular}{llcc}
\hline Rank & Country & Total citations & Average article citations \\
\hline 1 & USA & 63,009 & 15.808 \\
2 & China & 12,991 & 7.045 \\
3 & United Kingdom & 12,821 & 14.669 \\
4 & Italy & 8229 & 13.995 \\
5 & Korea & 6628 & 12.297 \\
6 & Germany & 5463 & 10.926 \\
7 & France & 3996 & 11.45 \\
8 & Canada & 3969 & 8.666 \\
9 & Netherlands & 3759 & 16.133 \\
10 & Australia & 3501 & 9.779 \\
11 & Japan & 3339 & 7.371 \\
12 & Spain & 2416 & 6.844 \\
13 & India & 2334 & 3.653 \\
14 & Switzerland & 2222 & 13.386 \\
15 & Sweden & 2011 & 15.007 \\
16 & Singapore & 1653 & 12.429 \\
17 & New Zealand & 1622 & 19.082 \\
18 & Belgium & 1493 & 15.883 \\
19 & Turkey & 1146 & 7.031 \\
20 & Slovenia & 1101 & 28.974 \\
\hline & & &
\end{tabular}

\subsection{Keywords Dynamics - Authors Versus Keywords Plus}

Table 5 provides the most frequent reference to the word dynamics, based on the author's keywords and keywords plus (not provided by authors, but found by the algorithm). Because of the keywords used in the search ("machine learning", "artificial intelligence", "deep learning", "learning", and, "healthcare"), it is clear that it appeared in the first positions on the author's side. However, it should be noted that "machine learning" reached virtually twice the number of frequency for "artificial intelligence". Also, we can observe the popularity of "robotics" and its variations. Other keywords also proved to have good performance. For instance, "big data", "data mining", "ethics", and "personalized medicine". Considering the keywords plus, approaches such as "classification", "outcomes", "system", "prediction", and "model", came out in the top 10, thus reinforcing the keywords provided by the authors. Moreover, in the keywords plus outlook, other important related topics emerged. They include "risk", "validation", "performance", etc. In this respect, this set of keywords have an important connection with AI techniques because it reflects some concerns related to risks, management, and performance. Thus, suggesting a connection and 
Table 4 Most global cited documents

\begin{tabular}{|c|c|c|c|c|c|}
\hline Rank & $\mathrm{AU}$ & TI & $\mathrm{SO}$ & $\mathrm{TC}$ & TCY \\
\hline 1 & $\begin{array}{l}\text { (Obermeyer } \\
\text { et al., 2016) }\end{array}$ & Predicting the Future - Big Data, Machine Learning, and Clinical Medicine & $\begin{array}{l}\text { New England Journal of } \\
\text { Medicine }\end{array}$ & 662 & 110.33 \\
\hline 2 & $\begin{array}{l}\text { (Giulianotti } \\
\quad \text { et al., 2003) }\end{array}$ & Robotics in general surgery - Personal experience in a large community hospital & Archives of Surgery & 591 & 31.11 \\
\hline 3 & (Topol 2019) & High-performance medicine: the convergence of human and artificial intelligence & Nature Medicine & 565 & 188.33 \\
\hline 4 & $\begin{array}{l}\text { (Xiong et al., } \\
\text { 2015) }\end{array}$ & The human splicing code reveals new insights into the genetic determinants of disease & Science & 540 & 77.14 \\
\hline 5 & (Majidi 2014) & Soft Robotics: A Perspective-Current Trends and Prospects for the Future & Soft Robotics & 532 & 66.50 \\
\hline 6 & $\begin{array}{l}\text { (Kononenko } \\
\text { 2001) }\end{array}$ & Machine learning for medical diagnosis: history, state of the art and perspective & $\begin{array}{l}\text { Artificial Intelligence in } \\
\text { Medicine }\end{array}$ & 508 & 24.19 \\
\hline 7 & $\begin{array}{l}\text { (De Fauw } \\
\text { et al., 2018) }\end{array}$ & Clinically applicable deep learning for diagnosis and referral in retinal disease & Nature & 478 & 119.50 \\
\hline 8 & $\begin{array}{l}\text { (Perry et al., } \\
\text { 2007) }\end{array}$ & Upper-Limb Powered Exoskeleton Design & $\begin{array}{l}\text { IEEE/ASME } \\
\text { Transactions on } \\
\text { Mechatronics }\end{array}$ & 474 & 31.60 \\
\hline 9 & $\begin{array}{l}\text { (Ahlering et al., } \\
\text { 2003) }\end{array}$ & $\begin{array}{l}\text { Successful transfer of open surgical skills to a laparoscopic environment using a } \\
\text { robotic interface: Initial experience with laparoscopic radical prostatectomy }\end{array}$ & Journal of Urology & 464 & 24.42 \\
\hline 10 & (Deo 2015) & Machine Learning in Medicine & Circulation & 463 & 66.14 \\
\hline 11 & $\begin{array}{l}\text { (Burke et al., } \\
\text { 2004) }\end{array}$ & The state of the art of nurse rostering & Journal of Scheduling & 457 & 25.39 \\
\hline 12 & $\begin{array}{l}\text { (Hu et al., } \\
\text { 2018) }\end{array}$ & Small-scale soft-bodied robot with multimodal locomotion & Nature & 396 & 99.00 \\
\hline 13 & $\begin{array}{l}\text { (Rajkomar } \\
\text { et al., } \\
2018 b)\end{array}$ & Scalable and accurate deep learning with electronic health records & NPJ Digital Medicine & 387 & 96.75 \\
\hline 14 & $\begin{array}{l}\text { (Ching et al., } \\
\text { 2018) }\end{array}$ & Opportunities and obstacles for deep learning in biology and medicine & $\begin{array}{l}\text { Journal of the Royal } \\
\text { Society Interface }\end{array}$ & 384 & 96.00 \\
\hline 15 & $\begin{array}{l}\text { (Mellit \& } \\
\text { Kalogirou } \\
\text { 2008) }\end{array}$ & Artificial intelligence techniques for photovoltaic applications: A review & $\begin{array}{l}\text { Progress in Energy and } \\
\text { Combustion Science }\end{array}$ & 376 & 26.86 \\
\hline 16 & $\begin{array}{l}\text { (Tewari et al., } \\
\text { 2003) }\end{array}$ & $\begin{array}{l}\text { A prospective comparison of radical retropubic and robot-assisted prostatectomy: ex- } \\
\text { perience in one institution }\end{array}$ & BJU International & 370 & 19.47 \\
\hline 17 & $\begin{array}{l}\text { (Benway et al., } \\
\text { 2009) }\end{array}$ & $\begin{array}{l}\text { Robot Assisted Partial Nephrectomy Versus Laparoscopic Partial Nephrectomy for } \\
\text { Renal Tumors: A Multi-Institutional Analysis of Perioperative Outcomes }\end{array}$ & Journal of Urology & 358 & 27.54 \\
\hline 18 & $\begin{array}{l}\text { (Aisen et al., } \\
\text { 1997) }\end{array}$ & $\begin{array}{l}\text { The effect of robot-assisted therapy and rehabilitative training on motor recovery } \\
\text { following stroke }\end{array}$ & Archives of Neurology & 355 & 14.20 \\
\hline 19 & $\begin{array}{l}\text { (Lei et al., } \\
\text { 2017) }\end{array}$ & $\begin{array}{l}\text { A Bioinspired Mineral Hydrogel as a Self-Healable, Mechanically Adaptable Ionic } \\
\text { Skin for Highly Sensitive Pressure Sensing }\end{array}$ & Advanced Materials & 345 & 69.00 \\
\hline 20 & $\begin{array}{l}\text { (Benight et al., } \\
\text { 2013) }\end{array}$ & Stretchable and self-healing polymers and devices for electronic skin & $\begin{array}{l}\text { Progress in Polymer } \\
\text { Science }\end{array}$ & 340 & 37.78 \\
\hline
\end{tabular}

Note: $\mathrm{AU}=$ Authors $\mathrm{TI}=\mathrm{Titl} ; \mathrm{SO}=$ Source $\mathrm{TC}=$ Total citation; $\mathrm{TCY}=$ Total citation per year

some concerns in the relationship between "Responsible AI" and "Ethical" practices in healthcare systems.

\subsubsection{Keywords Dynamics - Title-based TreeMap}

Considering the dynamics of the keywords but examining only the titles, we retrieved the most popular (see Fig. 3). The size of the rectangles is defined according to the word's frequency. Accordingly, on the left side, we have "learning", "machine", "artificial", "data", "intelligence", "deep", "robotassisted", and "system", as the most popular. Also, there is a clear emergence of important words related to the AI approach, such as "prediction", "robotic", "robot", "network", "detection", "model", among others. On the one hand, while a great diffusion of AI-related approaches in healthcare systems, on the other hand, titles regarding the interplay between "Responsible AI" and "ethical" issues is practically scarce. Therefore, this bolsters the need for a responsible AI in a digital health environment and an urgent investigation considering the society angle.

\subsection{Trend Topics}

Bearing in mind the AI emerging topics, Fig. 4 emphasizes the related dynamics and the prominent trends using a log frequency. In this regard, there appear various methods that 
Table 5 Most frequent words (Authors keywords versus keywords plus)

\begin{tabular}{lllll}
\hline Rank & Authors keywords & Occurrences & keywords plus & Occurrences \\
\hline 1 & machine learning & 2760 & classification & 940 \\
2 & artificial intelligence & 1619 & outcomes & 609 \\
3 & deep learning & 1139 & system & 594 \\
4 & learning & 475 & cancer & 585 \\
5 & healthcare & 462 & surgery & 577 \\
6 & robotics & 402 & prediction & 545 \\
7 & classification & 371 & diagnosis & 495 \\
8 & big data & 342 & risk & 493 \\
9 & machine & 323 & model & 406 \\
10 & robotic surgery & 289 & management & 399 \\
11 & data mining & 271 & care & 395 \\
12 & laparoscopy & 261 & experience & 339 \\
13 & precision medicine & 258 & validation & 316 \\
14 & ethics & 248 & medicine & 313 \\
15 & robot & 228 & impact & 309 \\
16 & medicine & 225 & mortality & 307 \\
17 & prediction & 205 & disease & 277 \\
18 & natural language processing & 191 & complications & 276 \\
19 & personalized medicine & 184 & big data & 265 \\
20 & artificial & 174 & performance & 256 \\
\hline & & & \\
\hline
\end{tabular}

emerged with consistent growth in the nineties ("neural networks" and expert systems") and were popularized and reinvigorated in the 2000s. In addition, emerging topics like "biomarkers" (to identify a person's health status), feature among those that could be discovered and analyzed by using different AI methods. Artificial neural networks seem to be one of the most popular AI techniques used in different healthcare approaches. Besides, there is a growing concern about the sensitivity of models and economic issues. Lastly, "expert system", "knowledge-based system", and "fuzzy-logic" remain a popular AI approach, but with less growth than other topics. However, the term "knowledge" isolated is one of the most popular topics. Similar to the TreeMap findings, "Responsible AI" and "ethical" issues have no direct participation in this type of analysis.

\subsection{Cluster Analysis}

To gain a more understanding of the dynamics of the topics, we performed a cluster analysis to identify topics with similar features (Kafeza et al., 2020; Kapoor et al., 2018). Accordingly, we considered the parameters highlighted in Table 6.

We found four clusters considering the whole of the period (1977-2020) (Fig. 5). The first (red) is a miscellaneous cluster but dominated by "artificial intelligence" approaches, including "big data", "simulation", "recognition", etc. Besides, other emerging technologies like "blockchain" and the "internet of things" belong to this cluster. Furthermore, the cluster integrates some topics related to responsible AI and ethical issues. For instance, "ethics", "privacy", "security", "healthcare", etc. In the second cluster (yellow), we have other AI-related approaches, like "deep learning", “classification", "support vector machines", "precision medicine", "bioinformatics", etc. In sequence, the third cluster (blue), predominated by "machine learning" and health issues, like "disease", "mortality", "epidemiology", "sepsis", etc. Finally, in the fourth cluster (green), we have the predominance of "robotics" approaches and "surgery", mainly applied in cancerous diseases.

In this vein, the findings suggest that AI approaches like "machine learning", "deep learning" were the most popular in the healthcare systems and, consequently, contributed to the field's digitalization. In addition, "big data", a popular technology used integrated with these AI technologies, was also an influential technique. Furthermore, emerging technologies like "blockchain" and "IoT" represent challenges and opportunities for responsible AI, ethics, privacy and security in a digital health system. For example, robots' usage is emerging a debate considering the role of "robot ethics" in surgery and other health activities.

\section{Discussion}

In this work, we explored AI's role in the transition of healthcare systems to a more digital health environment. 

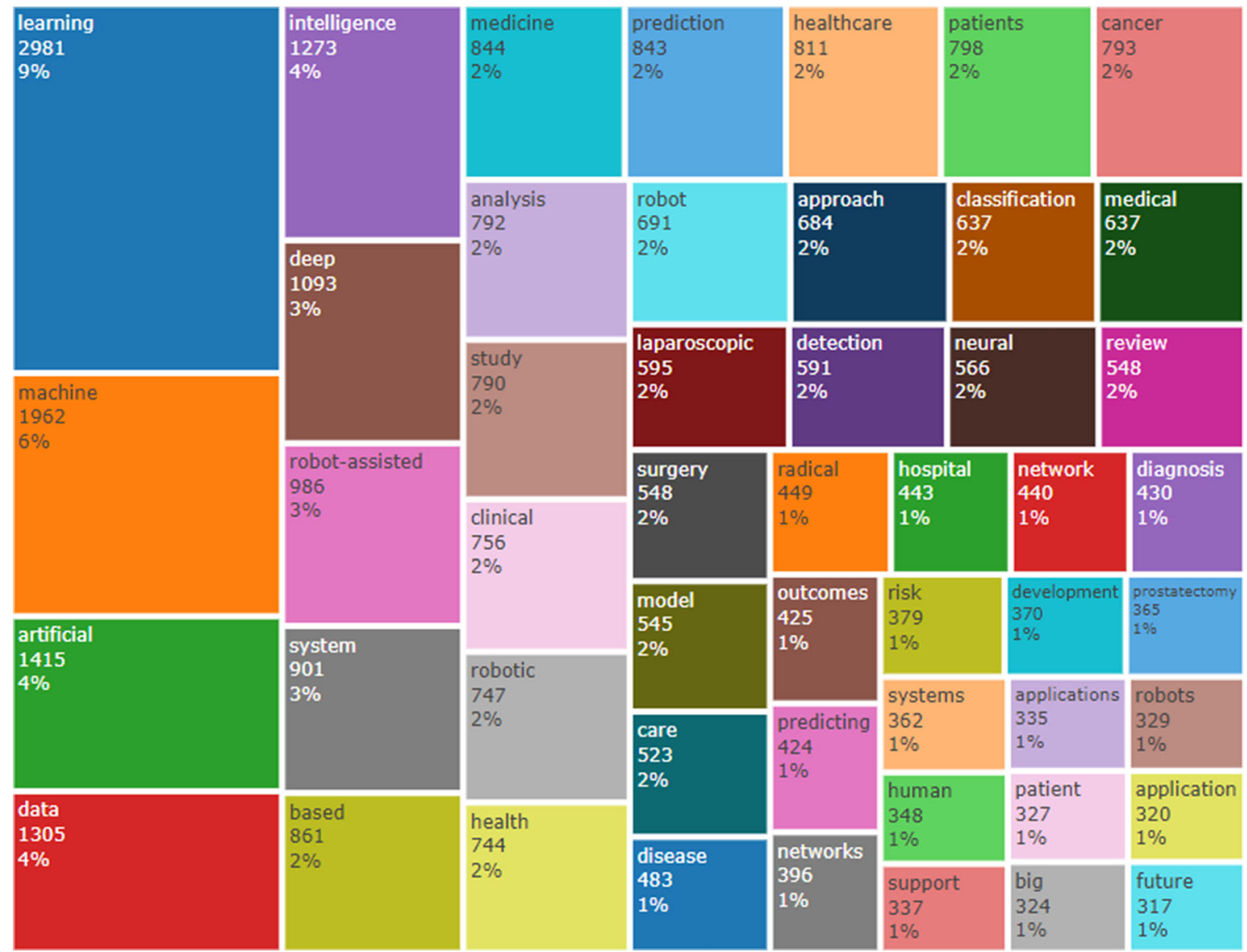

Fig. 3 TreeMap based on the abstracts

Comparing with the recent literature concerning AI topics by a literature review (Dwivedi et al., 2019; Fosso Wamba et al., 2021; Haefner et al., 2021), our findings are distinct due to this work being one of the first studies that investigate a considerable period covered by WoS database on the AI and responsible AI literature, considering the interplay with ethical issues on the healthcare field, and its behavior towards the digital health. Thus, in the next sections, we provide an in-depth discussion showing the advances in the emerging literature on responsible AI applied in digital health (Wang et al., 2020).

\subsection{Theoretical Contributions}

\subsubsection{What is the Publication Dynamics on the Interplay between $\mathrm{Al}$ and Healthcare Systems?}

Our bibliometric analysis presented the relevant productivity indicators of the field's relevant productivity indicators by taking into account the first question. We found that the first paper, according to the search used, appeared in 1977. In addition, the analysis showed the dynamics of production over four main periods. The early stage of the publication occurred between 1977 and 1990. In this period, a few papers were published each year. In the second period, 1991 to 2006, because of the computer's unprecedented advances, the field started a new degree, outperforming 100 papers for the first time in 2006. The third period, spanning the years 2007 to 2014, exceeded 100 publications each year, nearing 500 in 2014. We can see that the AI techniques reached exceptional performance in this interval, thanks to computational power groundbreaking. Finally, 2015-2020, the fourth period was characterized by extraordinary growth in the publications through the years, virtually reaching 3,000 papers in 2019 and 4,000 in 2020.

Also, our work found the most productive sources. In this vein, an open-access journal masters the top 20 ranks (IEEE Access). On the one hand, the medical informatics journals were the most popular in the ranking. On the other hand, journals focusing on other fields (i.e., decision sciences, business, management science, operations management, among others) were not identified in this rank. In terms of the number of citations, the USA dominated the chart, but European and Asian countries obtained an expressive ranking. Unfortunately, underrepresented countries, especially from Latin America and Africa, did not appear in the top 20.

AI publications clearly demonstrate the crucial role of $\mathrm{AI}$ in the transition from a traditional healthcare system to a digital health system, but this came out with thought-provoking 


\section{Trend Topics}

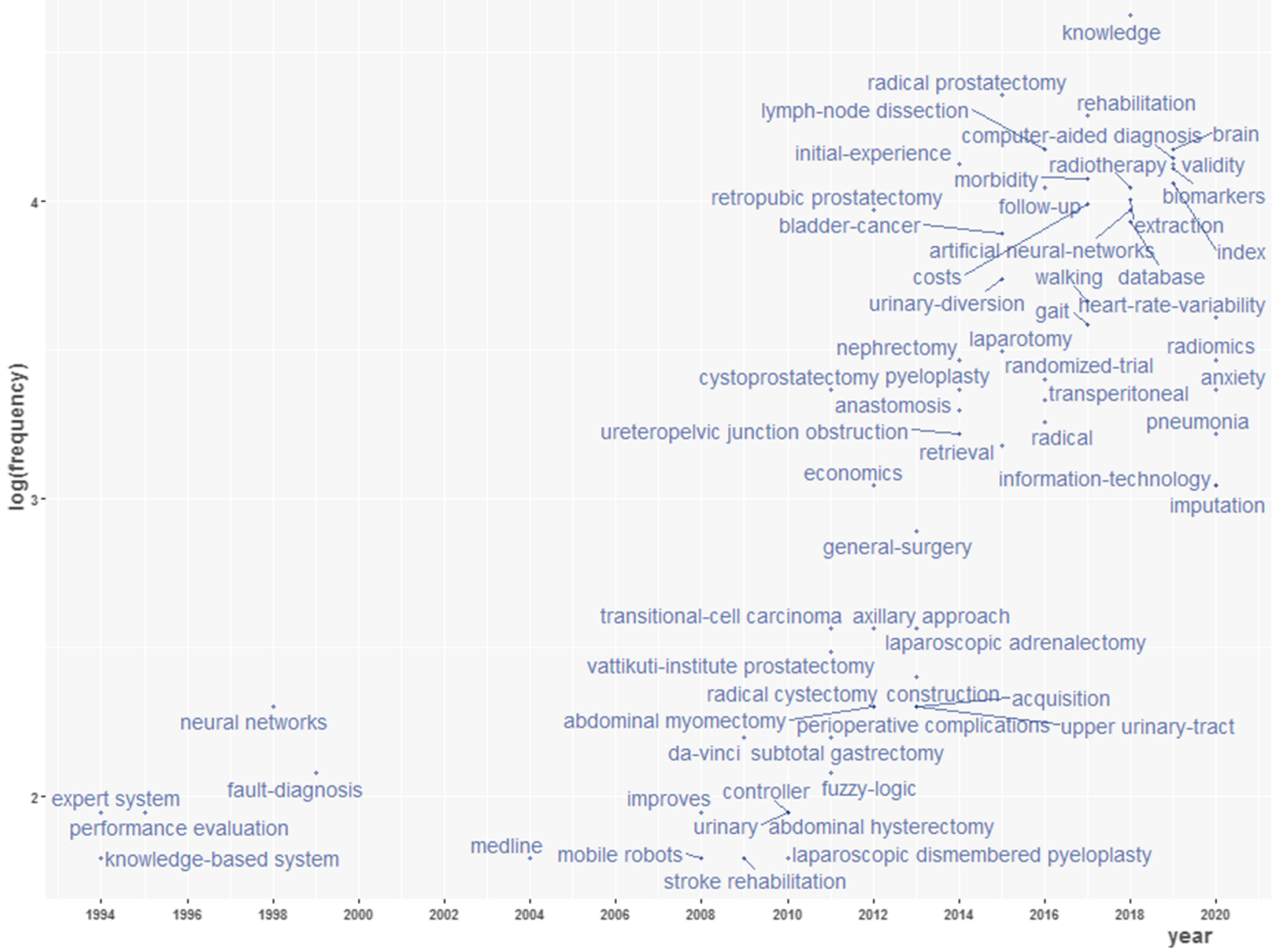

Fig. 4 Trend topics

issues. For instance, the majority of publications on the topic were made during the last five years (2015-2020), and the progress of healthcare systems was aligned with the computation breakthrough. The application of AI to healthcarerelated approaches was the predominant topic in medical informatics journals. Traditional journals are expected to follow this trend by integrating this important topic into their editorial objectives. Ultimately, universities from the underprivileged parts of the world should better embrace this topic, notably by developing partnerships with industrialized countries' hospitals, research institutions and governments.

\subsubsection{How is Artificial Intelligence Being Used in Digital Health Systems?}

In view of the results, our paper can draw some useful information considering how healthcare systems use artificial
Table 6 Cluster classification parameters

\begin{tabular}{ll}
\hline Type of analysis & Co-ocurrence \\
\hline Unit of analysis & All keywords (Author keywords and keywords plus) \\
Counting method & Full counting \\
Minimum number of a term & 50 \\
Meet the threshold & 243 \\
Clusters & 4
\end{tabular}




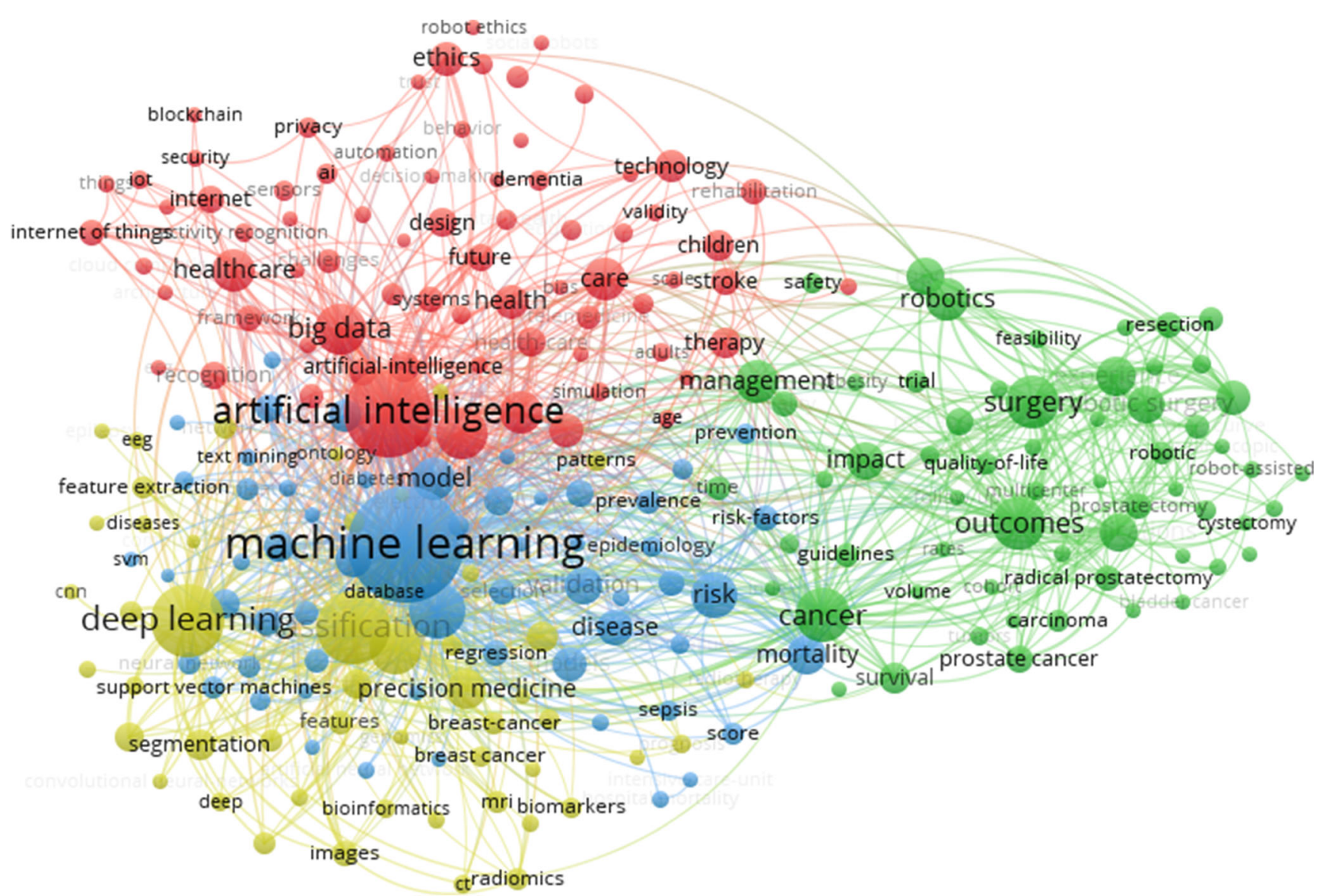

Fig. 5 Cluster classification papers 1977-2020

intelligence to proceed with their digitalization (Klinker et al., 2019; Lovis 2018; van Velthoven et al., 2019). From this perspective, Table 4 presented the most globally cited documents. According to the table, the robotics approach to support surgery activities gained momentum and created an environment where "robots" can assist patients with locomotion, which appears to be an important topic. Another popular approach consists in relying on machine learning, deep learning, and big data (that uses AI approaches) techniques to support medical diagnosis and prediction. Accordingly, a more personalized treatment can be provided for patients (Gottlieb et al., 2011; Rajkomar et al., 2019).

Moreover, by analyzing the dynamics of the keywords, our paper revealed that "machine learning", "deep learning" are some of the most common AI approaches. Big data techniques also demonstrated its importance for digital health systems, especially in the area of prediction and personalized medicine. Other emerging topics include "classification", "risk", "management", "model", "validation", and "performance". Finally, considering the dynamics of the keywords provided in the titles, we found that "learning", "machine", "artificial", "data", "intelligence", "deep", "robot-assisted", and "system" were the most popular topics. Not only does this reflect the advances achieved in the healthcare system's digitalization, but also it shows that the concerns about the use of technology are growing. However, despite such concerns about digital health systems, it is important to indicate a scarcity of responsible AI and ethical-related themes. But this is not the case with the issue of validation for which there are some concerns, together with a number of risks at the center stage of the debate. Thus, our results unlock and reinforce the urgent needs for more research on AI responsible regarding the ethical aspects of health systems and society's well-being (Wang et al., 2020).

\subsubsection{What are the Main Trends Regarding Artificial Intelligence in Digital Health Systems?}

With regard to the main trends of AI towards digital health systems, we can see AI approaches like machine learning, deep learning, artificial neural networks, expert systems, fuzzy logic, and convolutional neural network. Furthermore, other emerging technologies, like big data, blockchain and IoT, were identified as key technologies for supporting responsible $\mathrm{AI}$ in digital health (Dwivedi et al., 2019).

By considering the activities that robots can perform, it becomes obvious that these can well intervene in areas like surgery, rehabilitation, telemedicine, clinical trials, and 
hospital admissions, among others. Furthermore, AI support has given rise to other medical activities, including biomarkers, electronic medical records and big data for predictive models, recognition, monitoring, medical imaging and diagnosis, screening, early detection, prognosis and prediction, etc.

\subsubsection{What are the Main Limitations and Ethical Concerns About Responsible Artificial Intelligence Usage for Digital Health Systems?}

To answer this question, three categories of limitations were highlighted. First, privacy concerns (Ching et al., 2018) feature among the most relevant limitations/barriers to AI adoption and popularization in the healthcare systems. This adds to another leading privacy barrier, which is related to patient data (Sharma \& Kshetri 2020). The second category is associated with ethical issues - for example, regulations involving data acquisition and processing (Vayena et al., 2018). Besides, ethical governance and its implications for society (Wang et al., 2020) require a more in-depth debate. The third category is concerned with cultural resistance (Broadbent et al., 2009; Serrano et al., 2020; Wang et al., 2020). Therefore, these findings reinforce and advances the digital health literature (Wang et al., 2018), and more specifically, on responsible AI in health systems (Wang et al., 2020), considering the impact and importance of ethical issues (Vayena et al., 2018; Wearn et al., 2019).

\subsection{Theoretical Implications}

Recognizing our main findings related to AI technologies' role in turning healthcare systems into digital environments, Fig. 6 underlines a 4-level categorization of what can be expected: AI-related technologies, applications, benefits, and barriers.

With regard to leading AI technologies, the most popular approaches are machine learning and deep learning (Esteva et al., 2019; Rajkomar et al., 2019). These AI methods could be applied in different patient activities (e.g., medical diagnosis, telemedicine, exoskeleton rehabilitation, etc.). But we also found that surgery assistance, clinical trials, diagnosis, medical records, among others, are the most common AI application in digital health. Of the many benefits of digital health systems include personalized medicine (He et al., 2019), efficiency and agility positively impact the hospitals and the patients. In addition, AI techniques can improve accuracy in disease prediction (Jadhav et al., 2019), and thus the patient experience and well-being during their journey.

Concerning the different barriers to the medical sector's digitalization, several issues need to be adequately addressed and managed to achieve a more responsible AI in digital health systems. For instance, there are issues of patient's trustworthiness on the technologies (Vayena et al., 2018), ethics and data-related privacy (Vayena et al., 2018). Furthermore, established norms and government rules and regulations represent barriers in some respects, especially for hospitals and other health settings. Lastly, evidence has shown that resistance at the patients, medical and organizational levels represents a strong barrier to adopting and implementing digital health systems.

\subsubsection{Propositions Derived From the Framework}

Taking into account the proposed categorization (framework), it enables a set of propositions considering responsible $\mathrm{AI}$ and ethical concerns. In this regard, the interplay between AIrelated technologies and digital health should be anchored in strong ethical practices, information security, well-being society, workers skills, and organizations AI-culture. In this outlook, in Fig. 7 we introduce five insightful and challenging propositions.

Ethical practices In digital health, ethical concerns, especially related to patient sensitive information (Wang et al., 2018), and medical ethical tensions (He et al., 2019), can determine the responsible AI behavior in the healthcare systems. Thus, the following proposing emerges:

P1: Ethical practices impact the responsible AI towards digital health.

Information security Similarly to some ethical practices, information security, is a sensitive topic concerning AI towards digital health. It includes clear data governance, regulations, data processing restrictions, etc. (Vayena et al., 2018). Based on this, we propose the following proposition:

P2: Information security impact the responsible AI towards digital health.

Organization Al-culture Although AI-culture being an unexplored topic in the responsible AI literature, Wang et al. (2020) highlight that this is a critical aspect of supporting the responsible AI implementation. In this sense, a culture with strong data-driven practices, top management support and clear governance can leverage responsible AI projects in a digital health landscape (Sharma \& Kshetri 2020). Hence, we propose that:

P3: The organization AI-culture impact the responsible AI towards digital health.

Skillful workers The availability of talented workers is one of the critical (Dwivedi et al., 2019) aspects considering successful AI implementation projects. This behavior also occurs 
Fig. 6 Categorization of the main findings. *Usually, big data can combine different AI applications, and this is why, for this work, we integrated it into the "AI technologies" category

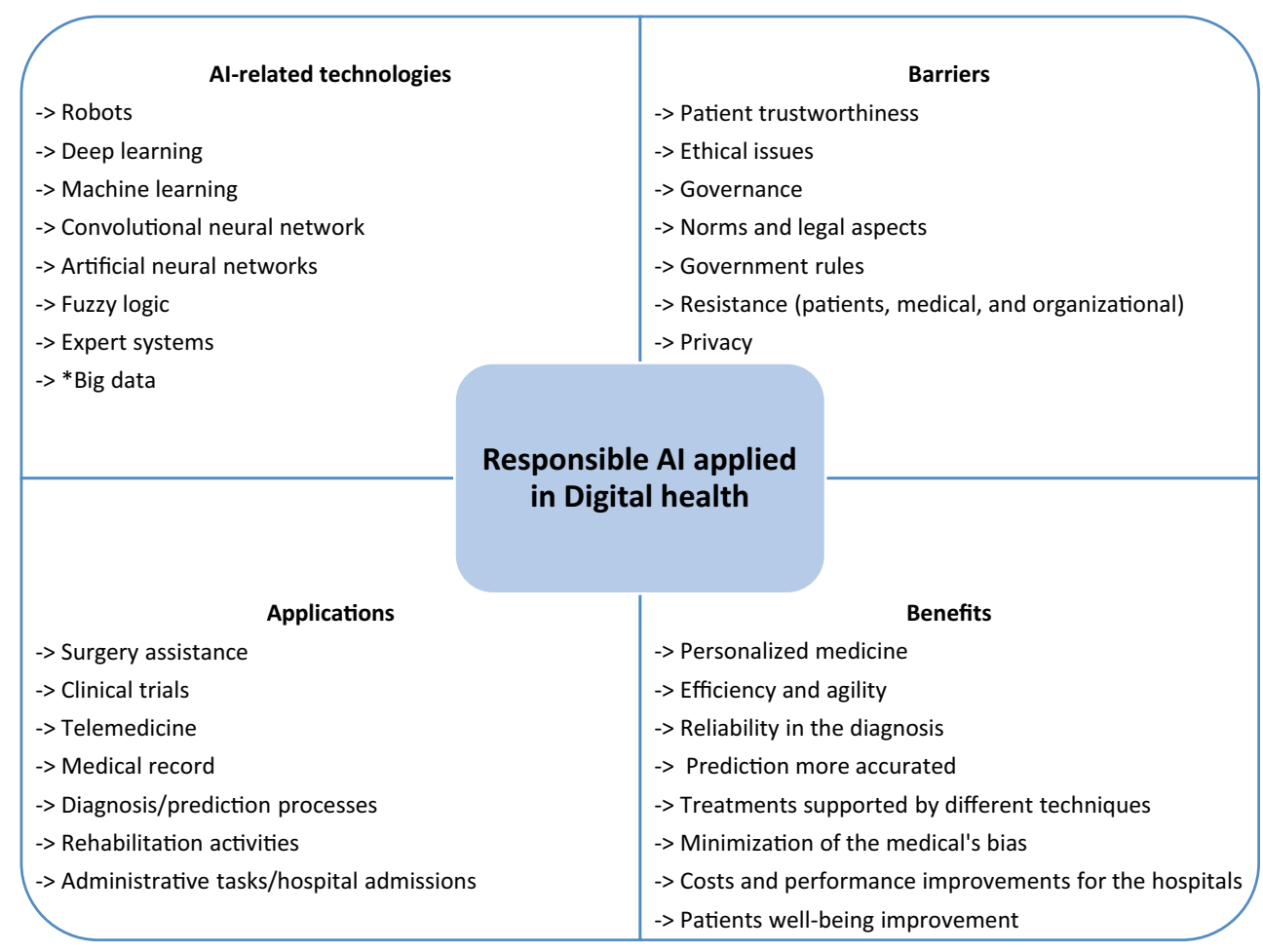

from a digital health perspective (Wang et al., 2020). Thus, the lack of skills (Serrano et al., 2020) can be a strong barrier regarding responsible $\mathrm{AI}$ in digital health. It impacts the implementation of AI projects, algorithms development, analysis and interpretation, among others. Accordingly, we derive the following proposition:

P4: The availability of skillful workers impact the responsible AI towards digital health.

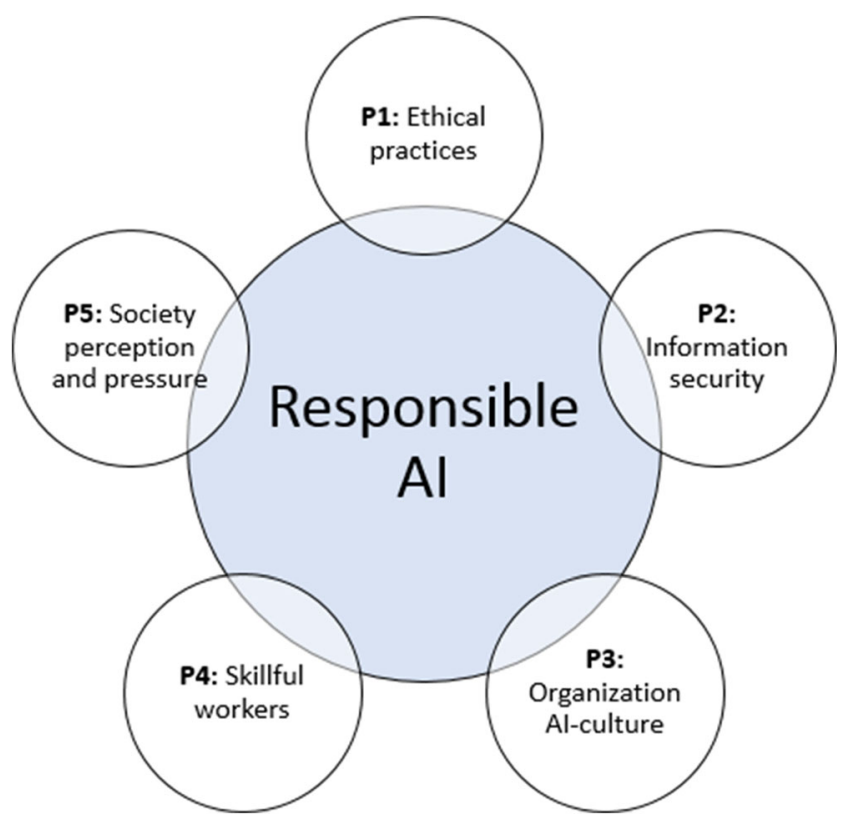

Fig. 7 Responsible AI propositions
Society perception and pressure One of the main objectives of AI usage is to leverage social well-being (Fosso Wamba et al., 2021). Considering the healthcare field, it is clear that AI can positively or negatively impact (Dwivedi et al., 2019). For instance, with telemedicine and smartphones, the process and costs of clinical trials could be improved, and the predictions for anticipate complex treatments. On the other side, the exposure of the patient's data, abusive "recommendations" for drugs and treatments, and medical tensions could negatively impact the responsible AI in digital health. In light of this, the following proposition emerges:

P5: The society pressure and perception impact the responsible AI towards digital health.

\subsubsection{Research Agenda and Directions}

Based on previous sections' findings, we proposed a research agenda and directions (Table 7) that may help scholars and practitioners to better understand and inquire about the interplay between $\mathrm{AI}$ and digital health and the prerequisites for responsible use of AI in healthcare environments.

The proposed agenda in digital health considers 11 main topics: (i) personalized medicine; (ii) telemedicine/telehealth; (iii) prediction; (iv) surgery; (v) admissions/administrative tasks; (vi) early detection and diagnosis; (vii) privacy issues; (viii) electronic health record ethics and challenges; (ix) governance models; (x) patients well-being; and xi. barriers to 
Table 7 Agenda and AI opportunities in digital health

\begin{tabular}{|c|c|c|}
\hline Main topics & Opportunities for future research & Related literature \\
\hline AI for personalized medicine & $\begin{array}{l}\text { Investigation of } \mathrm{AI} \text { techniques to provide a quick response and } \\
\text { personalized treatment } \\
\text { Exploration of } \mathrm{AI} \text { in conjunction with other related technologies to } \\
\text { improve the patient's journey }\end{array}$ & $\begin{array}{l}\text { (Rajkomar et al., 2019; Wang et al., 2020) } \\
\text { (Obermeyer et al., 2016; Wang et al., 2018) }\end{array}$ \\
\hline $\begin{array}{l}\text { AI applied in } \\
\text { telemedicine/telehealth }\end{array}$ & $\begin{array}{l}\text { Analyzing how devices and smart wearables could contribute to } \\
\text { telemedicine } \\
\text { Examination of barriers to telemedicine adoption }\end{array}$ & $\begin{array}{l}\text { (He et al., 2019; Wang et al., 2018) } \\
\text { (Serrano et al., 2020; Stephanie \& Sharma, 2020) }\end{array}$ \\
\hline AI for prediction & $\begin{array}{l}\text { Application of machine learning and deep learning techniques for } \\
\text { improving drug indication activities } \\
\text { Utilization of AI techniques to support disease and epidemic } \\
\text { outbreaks prediction }\end{array}$ & $\begin{array}{l}\text { (Chen \& Asch 2017; Ching et al., 2018; Gottlieb } \\
\text { et al., 2011; Jadhav et al., 2019) } \\
\text { (Dwivedi et al., 2019; Jadhav et al., 2019) }\end{array}$ \\
\hline AI for surgery & $\begin{array}{l}\text { Identification of the benefits and limitations of robots in healthcare } \\
\text { operations and therapy } \\
\text { Examination of the role in the human-robots interaction in surgery } \\
\text { operations }\end{array}$ & $\begin{array}{l}\text { (Broadbent et al., 2009; Esteva et al., 2019; Fasoli } \\
\text { et al., 2003) } \\
\text { (He et al., 2019; Majidi 2014) }\end{array}$ \\
\hline $\begin{array}{l}\text { AI for hospitals } \\
\text { admissions/administrative } \\
\text { tasks }\end{array}$ & $\begin{array}{l}\text { Investigating how efficiency and performance can be improved by } \\
\mathrm{AI} / \text { big data in administrative and operational activities } \\
\text { Investigating how AI techniques could contribute to minimizing } \\
\text { medical errors through enhanced information accuracy }\end{array}$ & $\begin{array}{l}\text { (Wang et al., 2018, 2020) } \\
\text { (He et al., 2019; Rajkomar et al., 2019) }\end{array}$ \\
\hline $\begin{array}{l}\text { AI for early detection and } \\
\text { diagnosis }\end{array}$ & $\begin{array}{l}\text { Exploring how AI could improve disease detection } \\
\text { The role of AI in clinical diagnosis and in supporting } \\
\text { underrepresented regions without adequate medical staff }\end{array}$ & $\begin{array}{l}\text { (He et al., 2019; Jiang et al., 2017) } \\
\text { (De Fauw et al., 2018; Jiang et al., 2017; } \\
\quad \text { Rajkomar et al., 2019) }\end{array}$ \\
\hline AI and health privacy issues & $\begin{array}{l}\text { Investigation of the best practices related to patient's data protection } \\
\text { Identification of the main privacy concerns in digital health systems }\end{array}$ & $\begin{array}{l}\text { (Jadhav et al., 2019; Wang et al., 2020) } \\
\text { (Klinker et al., 2019; Stephanie \& Sharma, 2020) }\end{array}$ \\
\hline $\begin{array}{l}\text { Electronic health record ethics } \\
\text { and issues }\end{array}$ & $\begin{array}{l}\text { Investigation of the main challenges and barriers related to } \\
\text { electronic health records } \\
\text { Identification of medical ethics tensions and the benefits of using } \\
\text { patient's health records }\end{array}$ & $\begin{array}{l}\text { (Rajkomar et al., 2018; Stephanie \& Sharma, } \\
\text { 2020) } \\
\text { (He et al., 2019) }\end{array}$ \\
\hline $\begin{array}{l}\text { Digital health governance } \\
\text { models }\end{array}$ & $\begin{array}{l}\text { Identification of challenges concerning AI ethical governance } \\
\text { practices }\end{array}$ & (Wang et al., 2020) \\
\hline $\begin{array}{l}\text { AI for improving patients } \\
\text { well-being }\end{array}$ & $\begin{array}{l}\text { Investigation of the role of responsible } \mathrm{AI} \text { in digital health and its } \\
\text { contribution to the patient's well-being }\end{array}$ & (Fosso Wamba et al., 2021; Wang et al., 2020) \\
\hline $\begin{array}{l}\text { Barriers related to AI adoption } \\
\text { in digital health systems }\end{array}$ & $\begin{array}{l}\text { Identification of barriers to the digitalization of health systems } \\
\text { transformation through AI }\end{array}$ & $\begin{array}{l}\text { (Dwivedi et al., 2019; Feldman et al., 2020; } \\
\text { Serrano et al., 2020; Sharma \& Kshetri 2020; } \\
\text { Stephanie \& Sharma, 2020) }\end{array}$ \\
\hline
\end{tabular}

adoption. For each topic, we highlighted some opportunities for further research by scholars and practitioners.

\subsection{Practical Implications}

In this work, essential implications emerged from the bibliometric analysis. First, we identified the most popular AI approaches and other emerging technologies (i.e., machine learning, deep learning, big data, blockchain, IoT, etc.) and how it's being used in the digital health field; thus, it could be considered by practitioners in their digital health projects. Second, by the proposed framework (Responsible AI applied in digital health), we provided four essential categories (AI-related technologies, applications, benefits, and barriers), that all involved in the digital health projects, and considering the role of responsible AI, should consider not only in the projects and implementation, but it can support the AI-culture and governance.

\subsection{Limitations}

Our work has two main limitations. First, this study employed only one database (Web of Science - WoS) to perform the keywords search. Thus, some documents may not have been retrieved in our search, impacting the analysis. Second, the keywords and the author's analysis can create an analysis bias. Therefore, to address these limitations, future studies could combine different databases (i.e., WoS and Scopus), integrate other keywords, and perform additional analysis types (i.e., metaanalysis). 


\section{Final Remarks and Conclusion}

In this work, we relied on a bibliometric analysis to investigate the role of responsible artificial intelligence in digital health systems. By exploring four complementary research questions, we identified the publication dynamics behind the interplay between AI and healthcare systems, how digital health systems take advantage of responsible artificial intelligence, the main trends regarding the integration of artificial intelligence into digital health systems, and the main barriers and ethical concerns about the use of artificial intelligence for health systems digitalization. Regarding the publication outcomes, we found four periods in which a consistent growth was identified from 2007. We noticed that the year 2015 marked the start of unprecedented growth, thanks to computation and other cutting-edge technologies. This topic appeared to be mastered by the 'medical informatics' journal. Based on the number of citations per country, the USA, China, and the UK ranked the top three. Unfortunately, the participation of underrepresented countries on this topic was not sufficient. Our study also discovered that robotics and prediction models were two of the most popular topics, supported by machine learning, deep learning, natural language processing, artificial neural networks, and big data techniques. We recognized that machine learning and deep learning were two of the most popular AI approaches from the trends perspective. In addition, artificial neural networks, expert systems, fuzzy logic, convolutional neural network, big data, blockchain, IoT, and robotics are also hot topics, which are being used for several activities like diagnosis, electronic medical record, prediction, image recognition, monitoring, screening, early detection, etc. Furthermore, the AI limitations and barriers for digital health systems were summarized in three primary categories: privacy concerns, ethical issues, and cultural resistance.

Our work makes essential contributions to the emerging literature on responsible AI, information systems and the healthcare system's journey towards a more digital health system. The main findings' categorization could be a useful framework for scholars, practitioners, and decision-makers involved in related fields to advance AI approaches' digital health systems. Besides, the propositions derived from the framework represent an avenue for future research to be empirically investigated and validated. It can also be complemented by the proposed agenda considering insightful AI opportunities and directions in digital health. Ultimately, the key lessons of this work suggest that practitioners and the stakeholders involved in digital health should pay attention to the technology per si and in a set of interactions supported by organizational AI-culture, talented personnel, and social perceptions pressures. Furthermore, we identified that AI responsible approaches in the digital health context have critical medical tensions, especially considering data privacy and governance. Considering these findings, our work provided essential elements to minimize the gap between theory and practice on the responsible $\mathrm{AI}$ domain.

\section{References}

Ahlering, T. E., Skarecky, D., Lee, D., \& Clayman, R. V. (2003). Successful transfer of open surgical skills to a laparoscopic environment using a robotic interface: Initial experience with laparoscopic radical prostatectomy. Journal of Urology, 170(5), 1738-1741. https://doi.org/10.1097/01.ju.0000092881.24608.5e.

Aisen, M. L., Krebs, H. I., Hogan, N., McDowell, F., \& Volpe, B. T. (1997). The effect of robot-assisted therapy and rehabilitative training on motor recovery following stroke. Archives of Neurology, 54(4), 443-446. https://doi.org/10.1001/archneur.1997. 00550160075019 .

Alahakoon, D., Nawaratne, R., Xu, Y., De Silva, D., Sivarajah, U., \& Gupta, B. (2020). Self-building artificial intelligence and machine learning to empower big data analytics in smart cities. Information Systems Frontiers. https://doi.org/10.1007/s10796-020-10056-x.

Aria, M., \& Cuccurullo, C. (2017). bibliometrix: An R-tool for comprehensive science mapping analysis. Journal of Informetrics, 11(4), 959-975. https://doi.org/10.1016/j.joi.2017.08.007.

Benight, S. J., Wang, C., Tok, J. B. H., \& Bao, Z. (2013). Stretchable and self-healing polymers and devices for electronic skin. Progress in Polymer Science, 38(12), 1961-1977. https://doi.org/10.1016/j. progpolymsci.2013.08.001.

Benway, B. M., Bhayani, S. B., Rogers, C. G., Dulabon, L. M., Patel, M. N., Lipkin, M., Wang, A. J., \& Stifelman, M. D. (2009). Robot assisted partial nephrectomy versus laparoscopic partial nephrectomy for renal tumors: a multi-institutional analysis of perioperative outcomes. Journal of Urology, 182(3), 866-872. https://doi.org/10. 1016/j.juro.2009.05.037.

Beydoun, G., Abedin, B., Merigó, J. M., \& Vera, M. (2019). Twenty years of information systems frontiers. Information Systems Frontiers, 21(2), 485-494. https://doi.org/10.1007/s10796-01909925-x.

Broadbent, E., Stafford, R., \& MacDonald, B. (2009). Acceptance of healthcare robots for the older population: Review and future directions. International Journal of Social Robotics, 1(4), 319-330. https://doi.org/10.1007/s12369-009-0030-6.

Burke, E. K., De Causmaecker, P., Vanden Berghe, G., \& Van Landeghem, H. (2004). The state of the art of nurse rostering. Journal of Scheduling, 7(6), 441-499. https://doi.org/10.1023/B: JOSH.0000046076.75950.0b.

Chen, J. H., \& Asch, S. M. (2017). Machine learning and prediction in medicine - Beyond the peak of inflated expectations. New England Journal of Medicine, 376(26), 2507-2509. https://doi.org/10.1056/ nejmp 1702071.

Ching, T., Himmelstein, D. S., Beaulieu-Jones, B. K., Kalinin, A. A., Do, B. T., Way, G. P., Ferrero, E., Agapow, P. M., Zietz, M., Hoffman, M. M., Xie, W., Rosen, G. L., Lengerich, B. J., Israeli, J., Lanchantin, J., Woloszynek, S., Carpenter, A. E., Shrikumar, A., Xu, J., ... Greene, C. S. (2018). Opportunities and obstacles for deep learning in biology and medicine. In Journal of the Royal Society Interface, 5, 141. https://doi.org/10.1098/rsif.2017.0387.

Clarivate Analytics. (2020). Web of Science. https://clarivate.com/ webofsciencegroup/solutions/web-of-science/. Accessed 20 Oct 2020. 
De Fauw, J., Ledsam, J. R., Romera-Paredes, B., Nikolov, S., Tomasev, N., Blackwell, S., Askham, H., Glorot, X., O’Donoghue, B., Visentin, D., van den Driessche, G., Lakshminarayanan, B., Meyer, C., Mackinder, F., Bouton, S., Ayoub, K., Chopra, R., King, D., Karthikesalingam, A., ... Ronneberger, O. (2018). Clinically applicable deep learning for diagnosis and referral in retinal disease. Nature Medicine, 24(9), 1342+. https://doi.org/10. 1038/s41591-018-0107-6.

Deo, R. C. (2015). Machine learning in medicine. Circulation, 132(20), 1920-1930. https://doi.org/10.1161/CIRCULATIONAHA.115. 001593

Duan, Y., Edwards, J. S., \& Dwivedi, Y. K. (2019). Artificial intelligence for decision making in the era of Big Data - evolution, challenges and research agenda. International Journal of Information Management, 48(January), 63-71. https://doi.org/10.1016/j. ijinfomgt.2019.01.021.

Dwivedi, Y. K., Hughes, L., Ismagilova, E., Aarts, G., Coombs, C., Crick, T., Duan, Y., Dwivedi, R., Edwards, J., Eirug, A., Galanos, V., Ilavarasan, P. V., Janssen, M., Jones, P., Kar, A. K., Kizgin, H., Kronemann, B., Lal, B., Lucini, B., ... Williams, M. D. (2019). Artificial Intelligence (AI): Multidisciplinary perspectives on emerging challenges, opportunities, and agenda for research, practice and policy. International Journal of Information Management, July, 101994. https://doi.org/10.1016/j.ijinfomgt.2019.08.002

Esteva, A., Robicquet, A., Ramsundar, B., Kuleshov, V., DePristo, M., Chou, K., Cui, C., Corrado, G., Thrun, S., \& Dean, J. (2019). A guide to deep learning in healthcare. Nature Medicine, 25(1), 24-29. https://doi.org/10.1038/s41591-018-0316-z.

Fasoli, S. E., Krebs, H. I., Stein, J., Frontera, W. R., \& Hogan, N. (2003). Effects of robotic therapy on motor impairment and recovery in chronic stroke. Archives of Physical Medicine and Rehabilitation, 84(4), 477-482. https://doi.org/10.1053/apmr.2003.50110.

Feldman, S. S., Hikmet, N., Modi, S., \& Schooley, B. (2020). Impact of provider prior use of HIE on system complexity, performance, patient care, quality and system concerns. Information Systems Frontiers. https://doi.org/10.1007/s10796-020-10064-x.

Fosso Wamba, S., Bawack, R. E., Guthrie, C., Queiroz, M. M., \& Carillo, K. D. A. (2021). Are we preparing for a good AI society? A bibliometric review and research agenda. Technological Forecasting \& Social Change, 164, 120482. https://doi.org/10. 1016/j.techfore.2020.120482.

Giulianotti, P. C., Coratti, A., Angelini, M., Sbrana, F., Cecconi, S., Balestracci, T., \& Caravaglios, G. (2003). Robotics in general surgery - Personal experience in a large community hospital. Archives of Surgery, 138(7), 777-784. https://doi.org/10.1001/archsurg.138. 7.777.

Gottlieb, A., Stein, G. Y., Ruppin, E., \& Sharan, R. (2011). PREDICT: a method for inferring novel drug indications with application to personalized medicine. Molecular Systems Biology, 7. https://doi.org/ 10.1038/msb.2011.26

Haefner, N., Wincent, J., Parida, V., \& Gassmann, O. (2021). Artificial intelligence and innovation management: A review, framework, and research agenda. Technological Forecasting and Social Change, 162(June 2020), 120392. https://doi.org/10.1016/j.techfore.2020. 120392.

He, J., Baxter, S. L., Xu, J., Xu, J., Zhou, X., \& Zhang, K. (2019). The practical implementation of artificial intelligence technologies in medicine. Nature Medicine, 25(1), 30-36. https://doi.org/10.1038/ s41591-018-0307-0.

Hu, W., Lum, G. Z., Mastrangeli, M., \& Sitti, M. (2018). Small-scale softbodied robot with multimodal locomotion. Nature, 554(7690), 8185. https://doi.org/10.1038/nature25443.

Jadhav, S., Kasar, R., Lade, N., Patil, M., \& Kolte, S. (2019). Disease prediction by machine learning from healthcare communities. International Journal of Scientific Research in Science and Technology, 29-35. https://doi.org/10.32628/ijsrst19633.
Jiang, F., Jiang, Y., Zhi, H., Dong, Y., Li, H., Ma, S., Wang, Y., Dong, Q., Shen, H., \& Wang, Y. (2017). Artificial intelligence in healthcare: Past, present and future. Stroke and Vascular Neurology, 2(4), 230-243. https://doi.org/10.1136/svn-2017000101.

Kafeza, E., Makris, C., Rompolas, G., \& Al-Obeidat, F. (2020). Behavioral and migration analysis of the dynamic customer relationships on twitter. Information Systems Frontiers. https://doi.org/10. 1007/s10796-020-10033-4.

Kapoor, K. K., Tamilmani, K., Rana, N. P., Patil, P., Dwivedi, Y. K., \& Nerur, S. (2018). Advances in social media research: past, present and future. Information Systems Frontiers, 20(3), 531-558. https:// doi.org/10.1007/s10796-017-9810-y.

Kim, K. C. (2014). Robotics in general surgery. Robotics in General Surgery, 138(July), 1-511. https://doi.org/10.1007/978-1-4614$8739-5$.

Klinker, K., Wiesche, M., \& Krcmar, H. (2019). Digital transformation in health care: augmented reality for hands-free service innovation. Information Systems Frontiers. https://doi.org/10.1007/s10796019-09937-7.

Kononenko, I. (2001). Machine learning for medical diagnosis: history, state of the art and perspective. Artificial Intelligence in Medicine, 23(1), 89-109. https://doi.org/10.1016/S0933-3657(01)00077-X.

Lei, Z., Wang, Q., Sun, S., Zhu, W., \& Wu, P. (2017). A bioinspired mineral hydrogel as a self-healable, mechanically adaptable ionic skin for highly sensitive pressure sensing. Advanced Materials, 29(22), 1700321. https://doi.org/10.1002/adma.201700321.

Lovis, C. (2018). Digital health: A science at crossroads. International Journal of Medical Informatics, 110(October 2017), 108-110. https://doi.org/10.1016/j.ijmedinf.2017.12.006.

Majidi, C. (2014). Soft robotics: a perspective-current trends and prospects for the future. Soft Robotics, 1(1), 5-11. https://doi.org/10. 1089/soro.2013.0001.

Mellit, A., \& Kalogirou, S. A. (2008). Artificial intelligence techniques for photovoltaic applications: A review. Progress in Energy and Combustion Science, 34(5), 574-632. https://doi.org/10.1016/j. pecs.2008.01.001.

Miotto, R., Wang, F., Wang, S., Jiang, X., \& Dudley, J. T. (2017). Deep learning for healthcare: Review, opportunities and challenges. Briefings in Bioinformatics, 19(6), 1236-1246. https://doi.org/10. 1093/bib/bbx044.

Mishra, D., Gunasekaran, A., Papadopoulos, T., \& Childe, S. J. (2018). Big Data and supply chain management: a review and bibliometric analysis. Annals of Operations Research, 270(1-2), 313-336. https://doi.org/10.1007/s10479-016-2236-y.

Mukherjee, S. (2020). Emerging frontiers in smart environment and healthcare - A Vision. Information Systems Frontiers, 22(1), 23 27. https://doi.org/10.1007/s10796-019-09965-3.

Mukherjee, U. K., \& Sinha, K. K. (2020). Robot-assisted surgical care delivery at a hospital: Policies for maximizing clinical outcome benefits and minimizing costs. Journal of Operations Management, 66(1-2), 227-256. https://doi.org/10.1002/joom.1058.

Nobre, G. C., \& Tavares, E. (2017). Scientific literature analysis on big data and internet of things applications on circular economy: a bibliometric study. Scientometrics, 111(1), 463-492. https://doi. org/10.1007/s11192-017-2281-6.

Obermeyer, Ziad, M. D., \& Emanuel, E. J. (2016). Predicting the future Big data, machine learning, and clinical medicine. New England Journal of Medicine, 375(13), 1212-1216. https://doi.org/10.1056/ NEJMp1606181.Predicting.

Peng, Y., Shi, J., Fantinato, M., \& Chen, J. (2017). A study on the author collaboration network in big data*. Information Systems Frontiers, 19(6), 1329-1342. https://doi.org/10.1007/s10796-017-9771-1.

Perry, J. C., Rosen, J., \& Burns, S. (2007). Upper-limb powered exoskeleton design. IEEE/ASME Transactions on Mechatronics, 12(4), 408-417. https://doi.org/10.1109/TMECH.2007.901934. 
Queiroz, M. M., Ivanov, D., Dolgui, A., \& Fosso Wamba, S. (2020b). Impacts of epidemic outbreaks on supply chains: mapping a research agenda amid the COVID-19 pandemic through a structured literature review. Annals of Operations Research, Forthcoming. https://doi.org/10.1007/s10479-020-03685-7.

Queiroz, M. M., Fosso Wamba, S., Machado, M. C., \& Telles, R. (2020a). Smart production systems drivers for business process management improvement: An integrative framework. Business Process Management Journal, 26(5), 1075-1092. https://doi.org/ 10.1108/BPMJ-03-2019-0134.

Rajkomar, A., Dean, J., \& Kohane, I. (2019). Machine learning in medicine. New England Journal of Medicine, 380(14), 1347-1358. https://doi.org/10.1056/nejmra1814259.

Rajkomar, A., Oren, E., Chen, K., Dai, A. M., Hajaj, N., Hardt, M., Liu, P. J., Liu, X., Marcus, J., Sun, M., Sundberg, P., Yee, H., Zhang, K., Zhang, Y., Flores, G., Duggan, G. E., Irvine, J., Le, Q., Litsch, K., ... Dean, J. (2018). Scalable and accurate deep learning with electronic health records. ArXiv, March, 1-10. https://doi.org/10.1038/ s41746-018-0029-1.

Serrano, A., Garcia-Guzman, J., Xydopoulos, G., \& Tarhini, A. (2020). Analysis of barriers to the deployment of health information systems: a stakeholder perspective. Information Systems Frontiers, 22(2), 455-474. https://doi.org/10.1007/s10796-018-9869-0.

Sharma, R., \& Kshetri, N. (2020). Digital healthcare: Historical development, applications, and future research directions. International Journal of Information Management, 53(March), 102105. https:// doi.org/10.1016/j.ijinfomgt.2020.102105.

Sivarajah, U., Kamal, M. M., Irani, Z., \& Weerakkody, V. (2017). Critical analysis of Big Data challenges and analytical methods. Journal of Business Research, 70, 263-286. https://doi.org/10.1016/j.jbusres. 2016.08.001.

Stephanie, L., \& Sharma, R. S. (2020). Digital health eco-systems: An epochal review of practice-oriented research. International Journal of Information Management, 53(January 2019), 102032. https://doi. org/10.1016/j.ijinfomgt.2019.10.017.

Tewari, A., Srivasatava, A., \& Menon, M. (2003). A prospective comparison of radical retropubic and robot-assisted prostatectomy: experience in one institution. BJU International, 92(3), 205-210. https://doi.org/10.1046/j.1464-410X.2003.04311.x.

Topol, E. J. (2019). High-performance medicine: the convergence of human and artificial intelligence. Nature Medicine, 25(1), 44-56. https://doi.org/10.1038/s41591-018-0300-7.

van Velthoven, M. H., Cordon, C., \& Challagalla, G. (2019). Digitization of healthcare organizations: The digital health landscape and information theory. International Journal of Medical Informatics, 124(January), 49-57. https://doi.org/10.1016/j.ijmedinf.2019.01. 007.

Vayena, E., Blasimme, A., \& Cohen, I. G. (2018). Machine learning in medicine: Addressing ethical challenges. PLoS Medicine, 15(11), 4 7. https://doi.org/10.1371/journal.pmed.1002689.

Wang, Y., Kung, L. A., \& Byrd, T. A. (2018). Big data analytics: Understanding its capabilities and potential benefits for healthcare organizations. Technological Forecasting and Social Change, 126, 3-13. https://doi.org/10.1016/j.techfore.2015.12.019.

Wang, Y., Xiong, M., \& Olya, H. (2020). Toward an Understanding of Responsible Artificial Intelligence Practices. Proceedings of the 53rd Hawaii International Conference on System Sciences, October 2019. https://doi.org/10.24251/hicss.2020.610.

Wearn, O. R., Freeman, R., \& Jacoby, D. M. P. (2019). Responsible AI for conservation. Nature Machine Intelligence, 1(2), 72-73. https:// doi.org/10.1038/s42256-019-0022-7.

Xiong, H. Y., Alipanahi, B., Lee, L. J., Bretschneider, H., Merico, D., Yuen, R. K. C., Hua, Y., Gueroussov, S., Najafabadi, H. S., Hughes, T. R., Morris, Q., Barash, Y., Krainer, A. R., Jojic, N., Scherer, S. W., Blencowe, B. J., \& Frey, B. J. (2015). The human splicing code reveals new insights into the genetic determinants of disease. Science, 347(6218). https://doi.org/10.1126/science.1254806.

Publisher's Note Springer Nature remains neutral with regard to jurisdictional claims in published maps and institutional affiliations.

Samuel Fosso Wamba is a Professor at Toulouse Business School, France, and Visiting Professor of Artificial Intelligence in Business at The University of Bradford, UK. He earned his Ph.D. in industrial engineering at the Polytechnic School of Montreal, Canada. He also holds two master's degrees: one MSc in mathematics from the University of Sherbrooke in Canada and another MSc in electronic commerce with a major in computer science from HEC Montreal, Canada. His current research focuses on business on the business value of I.T., interorganizational systems adoption, use and impacts, supply chain management, electronic commerce, blockchain, artificial intelligence for business, social media, business analytics, big data, and open data. His work has been published in several international conferences proceedings and in international journals, including the following: Academy of Management Journal, European Journal of Information Systems, International Journal of Production Economics, International Journal of Operations \& Production Management, International Journal of Production Research, Journal of Business Research, Electronic Markets, Technology Forecasting and Social Change, Information Systems Frontiers, and Production Planning \& Control. In 2017, he won the Best Paper Award by The Academy of Management Journal and by The Electronic Markets: The International Journal on Networked Business. He has been serving as a member on the editorial board of five international journals. Moreover, he is a CompTIA RFID+ Certified Professional and the Academic Co-Founder of RFID Academia. Apart from teaching and conducting research, he leads the newly created Artificial Intelligence \& Business Analytics Cluster (TBS). In one of his areas of research, he has been recently recognized as the most influential scholar in big data analytics and enterprises based on the number of published articles and the number of citations, and he features among the $2 \%$ of the most influential scholars in the Mendeley database, which includes 100,000 world topscientists. He is ranked among the ClarivateTMs' $1 \%$ most cited scholars in the world for 2020, according to the "Highly Cited ResearchersTM" list that identifies global research scientists with exceptional influence $3 / 4$ as reflected by the number of papers frequently cited by their peers. Furthermore, his current Google Scholar h-index is 50, with 10,714 citations by May 3, 2021.

Maciel M. Queiroz is a Professor at Paulista University and Mackenzie Presbyterian University. He earned his Ph.D. in Naval Architecture and Ocean Engineering at the Polytechnic School of the University of Sao Paulo, Brazil. His current research focuses on Digital supply chain capabilities, Industry 4.0, AI, blockchain, big data, and IoT. He has published papers in top-tier international journals, including: International Journal of Information Management, International Journal of Production Research, International Journal of Production Economics, Production Planning \& Control, Technological Forecasting and Social Change, International Journal of Logistics Management, Supply Chain Management, Annals of Operations Research, Science of the Total Environment, Benchmarking, and Business Process Management Journal, among others. Moreover, many of his research papers are being presented during important conferences and published in the corresponding Proceedings. Maciel has been also serving as a Guest Editor for the International Journal of Information Management, Production Planning \& Control, Annals of Operations Research, the International Journal of Operations \& Production Management, Industrial Management \& Data Systems, and the Journal of Global Information Management, among others. He co-chaired various conferences dealing with Artificial Intelligence, including AIS \& MCIS 2019 and IFIP WG8.6 2020. 